\title{
Multi-Volumetric Refocusing of Light Fields
}

This paper was downloaded from TechRxiv (https://www.techrxiv.org).

\section{LICENSE}

CC BY 4.0

SUBMISSION DATE / POSTED DATE

$21-05-2020$ / 29-05-2020

CITATION

Jayaweera, Sakila S.; Edussooriya, Chamira; Wijenayake, Chamith; Agathoklis, Pan; Bruton, Len (2020):

Multi-Volumetric Refocusing of Light Fields. TechRxiv. Preprint. https://doi.org/10.36227/techrxiv.12349835.v1

$\mathrm{DOI}$

10.36227/techrxiv.12349835.v1 


\title{
Multi-Volumetric Refocusing of Light Fields
}

\author{
Sakila S. Jayaweera, Chamira U. S. Edussooriya Member, IEEE, Chamith Wijenayake Member, IEEE, \\ Panajotis Agathoklis, Senior Member, IEEE, and Len T. Bruton, Life Fellow, IEEE
}

\begin{abstract}
Geometric information of scenes available with fourdimensional (4-D) light fields (LFs) pave the way for postcapture refocusing. Light filed refocusing methods proposed so far have been limited to a single planar or a volumetric region of a scene. In this paper, we demonstrate simultaneous refocusing of multiple volumetric regions in LFs. To this end, we employ a 4-D sparse finite-extent impulse response (FIR) filter consisting of multiple hyperfan-shaped passbands. We design the 4-D sparse FIR filter as an optimal filter in the least-squares sense. Experimental results confirm that the proposed filter provides $64 \%$ average reduction in computational complexity with negligible degradation in the fidelity of multi-volumetric refocused LFs compared to a 4-D nonsparse FIR filter.
\end{abstract}

Index Terms-Light fields, volumetric refocusing, multidimensional FIR filters, sparse filters, low complexity.

\section{INTRODUCTION}

A four-dimensional (4-D) light field (LF) captures both textural and geometrical information of a scene whereas a two-dimensional (2-D) image captures only the textural information [1], [2]. We can exploit the geometrical information available with LFs to accomplish novel tasks which are not possible with 2-D images, e.g., depth estimation [3]-[6] and occlusion suppression [7]-[12].

Post-capture refocusing is another novel task that can be achieved with LFs. $\mathrm{Ng}$ et al. [13] first demonstrated this feature employing a hand-held LF camera. They achieved refocusing by shifting and averaging the sub-aperture images (SAIs) of a LF. Furthermore, Ng [14] developed a computationally efficient algorithm using multi-dimensional fast Fourier transform algorithms. In [15], Fiss et al. employed depthadaptive splatting to archive refocusing of LFs. All of these approaches achieved refocusing for a narrow-depth range. In [16], Dansereau et al. demonstrate post-capture refocusing over a wide-depth range, which they denote as volumetric refocusing. They employed a 4-D linear filter having a hyperfanshaped passband to achieve volumetric refocusing. Dansereau et al. [17] and Premaratne et al. [18] employed similar 4-D linear hyperfan filters for LF denoising. In [19], Premaratne et al. proposed a 4-D sparse finite-extent impulse response (FIR) filter having a hyperfan-shaped passband, designed using the windowing technique and hard thresholding, for volumetric

S. S. Jayaweera and C. U. S. Edussooriya are with the Department of Electronic and Telecommunication Engineering, University of Moratuwa, Moratuwa 10400, Sri Lanka (e-mails: sakilaj@uom.lk; chamira@uom.lk).

C. Wijenayake is with the School of Information Technology and Electrical Engineering, University of Queensland, Brisbane, QLD 4072, Australia (email: c.wijenayake@uq.edu.au).

P. Agathoklis is with the Department of Electrical and Computer Engineering, University of Victoria, Victoria, BC V8W 2Y2, Canada (e-mail: panagath@ece.uvic.ca).

L. T. Bruton is with the Department of Electrical and Computer Engineering, University of Calgary, Calgary, AB T2N 1N4, Canada (e-mail: bruton@ucalgary.ca).

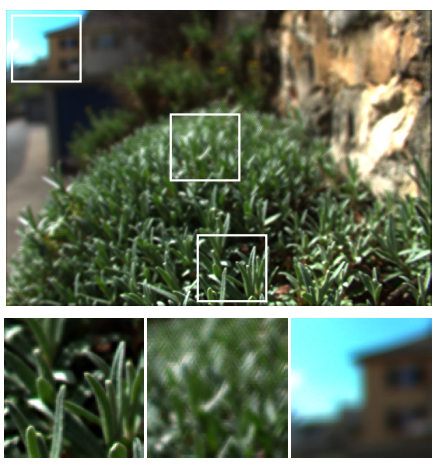

(a)

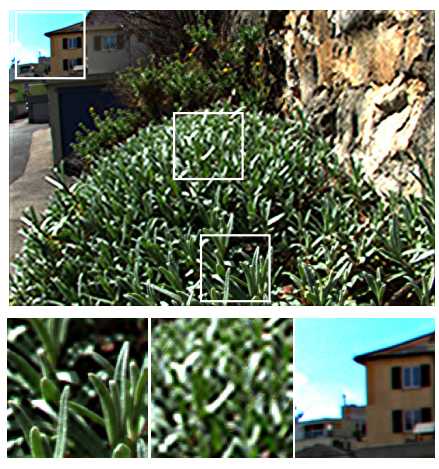

(b)
Fig. 1: Refocusing of the "Bush" LF; (a) refocused for a single volumetric region [19]; (b) refocused for two volumetric regions using the proposed 4-D sparse FIR filter.

refocusing. This sparse filter provides significant reduction in computational complexity with negligible degradation in the fidelity of refocused LFs compared to that proposed in [16]. However, all of these prior works are limited to LF refocusing over a single planar or a single volumetric region.

In this paper, we demonstrate simultaneous refocusing of multiple volumetric regions in LFs albeit at lower computational complexity. To this end, we employ a 4-D sparse FIR filter having multiple hyperfan-shaped passbands. Fig. 1 shows refocusing of a single volumetric region obtained with the 4-D sparse FIR filter proposed in [19] and simultaneous refocusing of two volumetric regions using the proposed 4D sparse FIR filter. It is evident that multi-volumetric refocusing can emphasize multiple objects or regions occupying different depth-ranges in an LF simultaneously. This feature may open new avenues in LF photography. In addition, our method can achieve refocusing of a single volumetric or a planar region as well because these are special cases of multi-volumetric refocusing. We design the 4-D sparse FIR filter by employing a two-step sparse filter design method proposed in [20], which considers the design of filters having quadrantally-symmetric impulse responses. Our 4-D sparse FIR filter has a centro-symmetric impulse response, and we adapt the two-step method in [20] appropriately to design our filter. Our 4-D sparse FIR filter is optimal in the leastsquares sense. Experimental results obtained with the LFs in the EPFL data set [21] confirm that the proposed 4-D sparse FIR filter provides $64 \%$ average reduction in computational complexity with negligible degradation in the fidelity of twovolumetric-region refocused LFs compared to an equivalent 4D non-sparse FIR filter. Furthermore, our sparse filter provides $13 \%$ reduction in computational complexity compared to that proposed in [19] with similar fidelity for refocusing of a single volumetric region. 


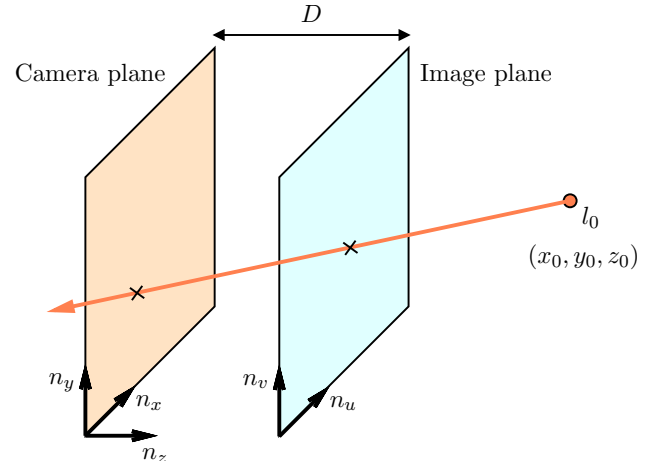

Fig. 2: Two plane parameterization of a Lambertian point source located at a constant depth $z_{0}$.

\section{REVIEW OF The SPECtRum OF A Light FiELD}

The spectrum of a LF is briefly reviewed in this section. To this end, we first consider standard two-plane paramaterization, with the globally defined image-plane coordinates, of a Lambertian point source as shown in Fig. 2. Note that $\left(n_{x}, n_{y}\right) \in \mathbb{Z}^{2}$ and $\left(n_{u}, n_{v}\right) \in \mathbb{Z}^{2}$ denote the 2-D discrete-domain camera-plane and image-plane coordinates, respectively, and $D$ is the constant distance between the camera and image planes. The 4-D LF $l_{p}(\mathbf{n})$, where $\mathbf{n}=$ $\left(n_{x}, n_{y}, n_{u}, n_{v}\right) \in \mathbb{Z}^{4}$, corresponding to the Lambertian point source located at $\left(x_{0}, y_{0}, z_{0}\right) \in \mathbb{R}^{2} \times \mathbb{R}^{+}$and having an intensity $l_{0}$ can be modeled as [22], [23]

$$
l_{p}(\mathbf{n})= \begin{cases}l_{0} \text { when } & \mathcal{P}_{x u} \equiv m n_{x} \Delta_{x}+n_{u} \Delta_{u}-(m+1) x_{0}=0 \\ & \mathcal{P}_{y v} \equiv m n_{y} \Delta_{y}+n_{v} \Delta_{v}-(m+1) y_{0}=0 \\ 0 & \text { elsewhere }\end{cases}
$$

where $m=\frac{D}{z_{0}}-1$ and $\Delta_{i}, i=x, y, u, v$ is the sampling interval along the dimension $i$. Note that the LF consists of a plane having a constant value $l_{0}$ given by the intersection two hyperplanes $P_{x u}$ and $P_{y v}$. In this case, the region of support (ROS) $\mathcal{R}_{p}$ of the spectrum $L_{p}(\boldsymbol{\omega})$, where $\omega=$ $\left(\omega_{x}, \omega_{y}, \omega_{u}, \omega_{v}\right) \in \mathbb{R}^{4}$, inside the principal Nyquist hypercube $\mathcal{N}\left(\triangleq\left\{\boldsymbol{\omega} \in \mathbb{R}^{4} \mid-\pi \leq \omega_{i}<\pi, i=x, y, u, v\right\}\right)$ is given by $\mathcal{R}_{p}=\mathcal{H}_{x u} \cap \mathcal{H}_{y v}$ [22], [23], where

$$
\begin{aligned}
\mathcal{H}_{x u} & =\left\{\boldsymbol{\omega} \in \mathbb{R}^{4} \mid \omega_{x}-\left(\frac{m \Delta_{x}}{\Delta_{u}}\right) \omega_{u}=0\right\} \\
\mathcal{H}_{y v} & =\left\{\boldsymbol{\omega} \in \mathbb{R}^{4} \mid \omega_{y}-\left(\frac{m \Delta_{y}}{\Delta_{v}}\right) \omega_{v}=0\right\}
\end{aligned}
$$

The $\operatorname{ROS} \mathcal{R}_{p}$ is a plane through the origin of $\boldsymbol{\omega}$ inside $\mathcal{N}$, of which the orientation depends only on the depth $z_{0}$ of the Lambertian point source. Note that we do not consider the finite sizes of the camera and image planes in presenting the spectral ROS for simplicity. However, even with these constraints, the spectral ROS predominantly occupies the region defined by the $\operatorname{ROS} \mathcal{R}_{p}$ [24], [25].

In order to obtain the ROS $\mathcal{R}_{o}$ of the spectrum of a LF corresponding to a Lambertian object, we can consider the Lambertian object as collection of Lambertian point sources located in a volumetric region with a depth range $z_{0} \in\left[d_{\min }, d_{\max }\right]$. In this case, $\mathcal{R}_{o}$ is given by $\mathcal{R}_{o}=\bigcup \mathcal{R}_{p}=\bigcup\left(\mathcal{H}_{x u} \cap \mathcal{H}_{y v}\right)$ [10], [22], and corresponds to a hyperfan inside $\mathcal{z _ { 0 }} \mathcal{N}$ [16], as shown in

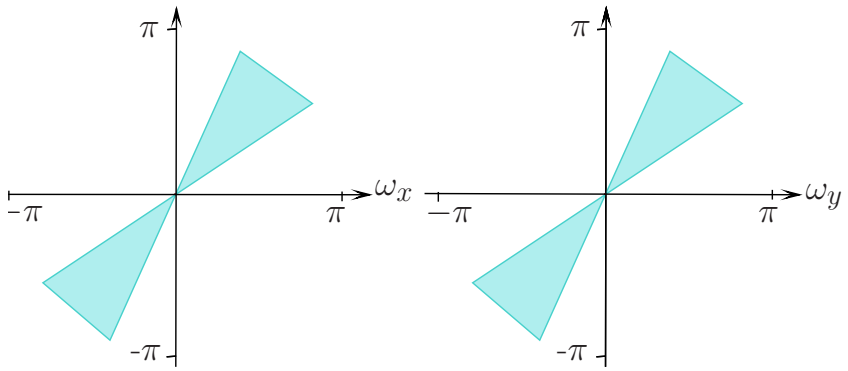

Fig. 3: The hyperfan-shaped spectral ROS $\mathcal{R}_{o}$ corresponding to a Lambertian object; (a) in the $\omega_{x} \omega_{u}$ subspace (b) in the $\omega_{y} \omega_{v}$ subspace.

Fig. 3. In the case of multiple objects located in $M$ volumetric regions, the spectral ROS $\mathcal{R}_{O}^{M}$ can be obtained as

$$
\mathcal{R}_{o}^{M}=\bigcup_{i=1}^{M} \mathcal{R}_{o}^{i}=\bigcup_{i=1}^{M} \bigcup_{z_{0}^{i}}\left(\mathcal{H}_{x u}^{i} \cap \mathcal{H}_{y v}^{i}\right) \text {. }
$$

The spectral $\operatorname{ROS} \mathcal{R}_{o}^{M}$ thus contains $M$ hyperfans inside $\mathcal{N}$. Therefore, we can refocus $M$ volumetric regions in a LF simultaneously by employing a 4-D filter having $M$ hyperfanshaped passbands inside $\mathcal{N}$.

\section{Proposed 4-D SpARse FIR Filter}

The proposed 4-D sparse FIR filter $H(\mathbf{z}),\left(z_{x}, z_{y}, z_{u}, z_{v}\right) \in$ $\mathbb{C}^{4}$, is designed as a cascade of two 2-D filters $H_{x u}\left(z_{x}, z_{u}\right)$, $\left(z_{x}, z_{u}\right) \in \mathbb{C}^{2}$ and $H_{y v}\left(z_{y}, z_{v}\right),\left(z_{y}, z_{v}\right) \in \mathbb{C}^{2}$, as shown in Fig. 4. This structure is motivated by the partial separability of the spectral ROS of a Lambertian point source and leads to an extremely low computational complexity of $O\left(N_{x} N_{u}+N_{y} N_{v}\right)$ to process a sample, compared to that of a nonseparable 4-D filter, which is $O\left(N_{x} N_{y} N_{u} N_{v}\right)$ [19], where $\left(N_{x}, N_{u}\right)$ $\left(\in \mathbb{Z}_{+}^{2}\right)$ and $\left(N_{y}, N_{v}\right)\left(\in \mathbb{Z}_{+}^{2}\right)$ are the orders of $H_{x u}\left(z_{x}, z_{u}\right)$ and $H_{y v}\left(z_{y}, z_{v}\right)$, respectively. We select the passbands of $H_{x u}\left(z_{x}, z_{u}\right)$ and $H_{y v}\left(z_{y}, z_{v}\right)$ to enclose $M$ hyperfan regions $\mathcal{B}_{x u}=\bigcup_{i=1}^{M} \bigcup_{z_{0}^{i}} \mathcal{H}_{x u}^{i}$ and $\mathcal{B}_{y v}=\bigcup_{i=1}^{M} \bigcup_{z_{0}^{i}} \mathcal{H}_{y v}^{i}$, respectively. Note that the passband of $\mathcal{B}$ of the 4-D FIR filter $H(\mathbf{z})$ given by $\mathcal{B}_{x u} \cap \mathcal{B}_{y v}$ completely encompasses the spectral ROS given by (2). Fig. 5 shows the passband of $H_{x u}\left(z_{x}, z_{u}\right)$ for $M=2$. Our 4-D FIR filter $H(\mathbf{z})$ sharpens (i.e., focuses) the depth ranges in a $\mathrm{LF}$, which corresponds to these $M$ hyperfans and blurs other depth ranges, which correspond to the stopband.

Fig. 5 shows the parameters that specify the $i$ th hyperfan of the passband of $H_{x u}\left(z_{x}, z_{u}\right)$. Here, $\alpha_{i}, \theta_{i}$ and $B_{i}$ determine the orientation, angular-width and the length of the $i$ th hyperfan, respectively, and $T_{i}$ determines the width of the guard band employed to achieve an improved accuracy near the origin of $\boldsymbol{\omega}$ [26]. The passband and the specifications of the $i$ th hyperfan is the same for $H_{y v}\left(z_{y}, z_{v}\right)$ in the $\omega_{y} \omega_{v}$ subspace. Therefore, the design of $H_{x u}\left(z_{x}, z_{u}\right)$ and $H_{y v}\left(z_{y}, z_{v}\right)$ reduces to a single 2-D FIR filter design. We next present the design of $H_{x u}\left(z_{x}, z_{u}\right)$ in detail.

\section{A. Weighted Least-Square Design of $H_{x u}\left(z_{x}, z_{u}\right)$}

We adapt the 2-D sparse FIR filter design method proposed in [20] to design $H_{x u}\left(z_{x}, z_{u}\right)$. To this end, we express the 


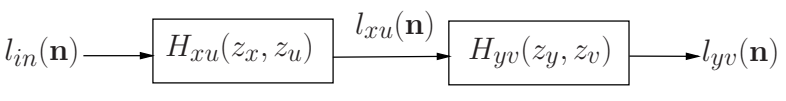

Fig. 4: The structure of the proposed 4-D sparse FIR filter.

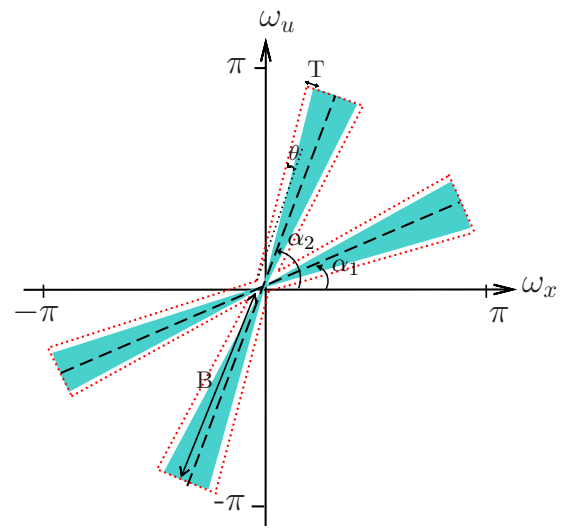

Fig. 5: The passband of $H_{x u}\left(z_{x}, z_{u}\right)$ for $M=2$.

frequency response of $H_{x u}\left(z_{x}, z_{u}\right)$ as $H_{x u}\left(e^{j \omega_{x}}, e^{j \omega_{u}}\right)=$ $\sum_{n_{x}=\frac{-N_{x}}{2}}^{\frac{N_{x}}{2}} \sum_{n_{u}=\frac{-N_{u}}{2}}^{\frac{N_{u}}{2}} h_{x u}\left(n_{x}, n_{u}\right) e^{-j\left(n_{x} \omega_{x}+n_{u} \omega_{u}\right)}$, where $h_{x u}\left(n_{x}, n_{u}\right)$ is the impulse response of size $\left(N_{x}+1\right) \times\left(N_{u}+1\right)$ (order $=N_{x} \times N_{u}$ ). We design $H_{x u}\left(z_{x}, z_{u}\right)$ as a zero-phase filter [27, ch.3]. In this case, the impulse response of the filter is centro-symmetric, i.e., $h_{x u}\left(n_{x}, n_{u}\right)=h_{x u}\left(-n_{x},-n_{u}\right)$. Therefore, we can simplify $H_{x u}\left(e^{j \omega_{x}}, e^{j \omega_{u}}\right)$ considering the centro-symmetric property as $H_{x u}\left(e^{j \omega_{x}}, e^{j \omega_{u}}\right)=h_{x u}(0,0)+$ $\sum_{n_{x}=1}^{\frac{N_{x}}{2}} 2 h_{x u}\left(n_{x}, 0\right) \cos \left(n_{x} \omega_{x}\right)+\sum_{n_{x}=\frac{N_{x}}{2}}^{\frac{N_{x}}{2}} \sum_{n_{u}=1}^{\frac{N_{y}}{2}} 2 h_{x u}\left(n_{x}, n_{u}\right)$ $\cos \left(n_{x} \omega_{x}+n_{u} \omega_{u}\right)$, which we represent in the vector-form as

$$
H_{x u}\left(e^{j \omega_{x}}, e^{j \omega_{u}}\right)=\boldsymbol{c}\left(\omega_{x}, \omega_{u}\right)^{\mathrm{T}} \boldsymbol{h}_{x u} .
$$

Here, $\quad \boldsymbol{h}_{x u}=\left[h_{x u}(0,0), 2 h_{x u}(1,0), \cdots, 2 h_{x u}\left(\frac{N_{x}}{2}, 1\right)\right.$, $\left.2 h_{x u}\left(\frac{-N_{x}}{2}, 1\right), \cdots, 2 h\left(\frac{N_{x}}{2}, \frac{N_{u}}{2}\right)\right]^{\mathrm{T}}$, and $\boldsymbol{c}\left(\omega_{x}, \omega_{u}\right)=\left[1, \cos \left(\omega_{x}\right)\right.$, $\left.\cdots, \cos \left(\frac{N_{x}}{2} \omega_{x}\right), \cos \left(\frac{-N_{x}}{2} \omega_{x}+\omega_{u}\right), \cdots, \cos \left(\frac{N_{x}}{2} \omega_{x}+\frac{N_{x}}{2} \omega_{u}\right)\right]^{\mathrm{T}}$.

$\mathrm{Lu}$ and Hinamoto proposed two-step weighted least-square approach in [20] to design 2-D sparse FIR filter having quadrantally-symmetric impulse responses. With (3), we can use this approach to design $H_{x u}\left(z_{x}, z_{u}\right)$ despite its centrosymmetric impulse response. In the first step, we obtain an intermediate sparse impulse response in the least-squares sense. We express the objective function $J\left(\boldsymbol{h}_{x u}\right)$ to be minimized as

$$
\begin{aligned}
& J\left(\boldsymbol{h}_{x u}\right)=\left[\int \int _ { \mathcal { F } } W ( \omega _ { x } , \omega _ { u } ) \left[H_{x u}^{I}\left(e^{j \omega_{x}}, e^{j \omega_{u}}\right)\right.\right. \\
& \left.\left.-H_{x u}\left(e^{j \omega_{x}}, e^{j \omega_{u}}\right)\right]^{2} \mathrm{~d} \omega_{x} \mathrm{~d} \omega_{u}\right]^{\frac{1}{2}}+\mu\left\|\boldsymbol{h}_{x u}\right\|_{1},
\end{aligned}
$$

where $H_{x u}^{I}\left(e^{j \omega_{x}}, e^{j \omega_{u}}\right)$ is the ideal frequency response of $H_{x u}\left(z_{x}, z_{u}\right)$ having 1 in the passband and 0 in the stopband, $H_{x u}\left(e^{j \omega_{x}}, e^{j \omega_{u}}\right)$ is given in (3), $W\left(\omega_{x}, \omega_{u}\right)$ is a weighting function that we use to control the stopband attenuation, $\mu$ is a small positive number (typically between 0.01 and 1 ), and $\mathcal{F}$ is the region corresponding to the passband and stopband, i.e. without the transition band [20]. Because, $H_{x u}\left(e^{j \omega_{x}}, e^{j \omega_{u}}\right)$ is centro-symmetric, we consider only the region $[-\pi, \pi] \times[0, \pi]$ in the 2-D frequency domain $\left(\omega_{x}, \omega_{u}\right)$ to define $\mathcal{F}$. By considering finite set of frequency grid points in $\mathcal{F}$ and introducing upper bounds for the first and second terms in the right hand side of (4), we can convert the optimization probelem as an $\ell_{1}-\ell_{2}$ minimization problem, which can be converted as a second-order cone programming problem [20]. Due to the limited space, we do not present the detailed steps, and the reader is referred to [20]. The solution $\boldsymbol{h}_{x u}^{i}$ of the secondorder cone programming problem is an approximately sparse impulse response [20], and we employ hard thresholding in order to obtain a sparse impulse response $\boldsymbol{h}_{x u}^{i, s}$, i.e.,

$$
\boldsymbol{h}_{x u}^{i, s}(i)= \begin{cases}\boldsymbol{h}_{x u}^{i}(i), & \text { if }\left|\boldsymbol{h}_{x u}^{i},(i)\right| \geq \epsilon_{t h} \\ 0, & \text { otherwise, }\end{cases}
$$

where $\epsilon_{t h}\left(\in\left[10^{-4}, 10^{-2}\right]\right.$, typically) is the threshold value.

In the second step, we again optimize $\boldsymbol{h}_{x u}^{i, s}$ in the leastsquares sense in order to further improve the accuracy. We express this optimization problem as

$$
\begin{aligned}
& \min _{\boldsymbol{h}_{x u}^{i, s}}\left[\int \int _ { \mathcal { F } } W ( \omega _ { x } , \omega _ { u } ) \left[\boldsymbol{c}\left(\omega_{x}, \omega_{u}\right)^{\mathrm{T}} \boldsymbol{h}_{x u}^{i, s}-\right.\right. \\
& \left.\left.H_{x u}^{I}\left(e^{j \omega_{x}}, e^{j \omega_{u}}\right)\right]^{2} \mathrm{~d} \omega_{x} \mathrm{~d} \omega_{u}\right]^{\frac{1}{2}} \\
& \text { subject to: } \quad \boldsymbol{h}_{x u}^{i, s}(i)=0 \text { for } i \in I_{\infty},
\end{aligned}
$$

where $I_{\infty}$ is the set containing indices $i$ for which $\boldsymbol{h}_{x u}^{i, s}(i)=0$. This optimization probelem is a quadratic program. We obtain the sparse impulse response $\boldsymbol{h}_{x u}^{s}$ as $\boldsymbol{h}_{x u}^{s}=\gamma \boldsymbol{h}_{x u}^{q, s}$, where $\boldsymbol{h}_{x u}^{q, s}$ is the solution of $(6)$, and $\gamma(\in[1,1.5]$, typically $)$ is a constant used to compensate the intensity reduction of a refocused LF due to the small number of SAIs.

\section{EXPERIMENTAL RESULTS}

In this section, we present the experimental results obtained for LFs in the EPFL dataset [21] for $M=2$ in the next subsection and for $M=3$ in supplementary results ${ }^{1}$. Furthermore, we compare the performance of the the proposed filter compared to a 4-D nonsparse FIR filter in multi-volumetric refocusing. Next, we compare the performance and computational complexity of the proposed filter with those of [16] and [19].

\section{A. Performance of the Proposed 4-D Sparse FIR Filter in Multi-Volumetric Refocusing}

We process five LFs, "Parc du Luxembourg", "Bush", "Books", "Sphynx", and "University", using the proposed 4D sparse FIR filter and a 4-D nonsparse FIR filter. Here, we select only the middle $11 \times 11$ SAIs for each LF and discard SAIs affected by vignetting. For the "Parc du Luxembourg" LF, we design $H_{x u}\left(z_{x}, z_{u}\right)$ and $H_{y v}\left(z_{y}, z_{v}\right)$ with $\alpha_{1}=50^{\circ}$ and $\alpha_{2}=120^{\circ}, \theta_{1}, \theta_{2}=10^{\circ}, B_{1}, B_{2}=0.9 \pi \mathrm{rad} / \mathrm{sample}$, $T_{1}, T_{2}=0.08 \pi \mathrm{rad} / \mathrm{sample}, \mu=0.1, \epsilon_{t h}=0.004, \gamma=1.4$, and $W\left(\omega_{x}, \omega_{u}\right)=1$ for the passband and $W\left(\omega_{x}, \omega_{u}\right)=2$ for the stopband. We present the specifications of $H_{x u}\left(z_{x}, z_{u}\right)$ or $H_{y v}\left(z_{y}, z_{v}\right)$ employed for the other four LFs in the supplementary results ${ }^{1}$. We select the order of $H_{x u}\left(z_{x}, z_{u}\right)$ and

\footnotetext{
${ }^{1}$ The supplementary results are available at https://bit.ly/2KP3F1k.
} 


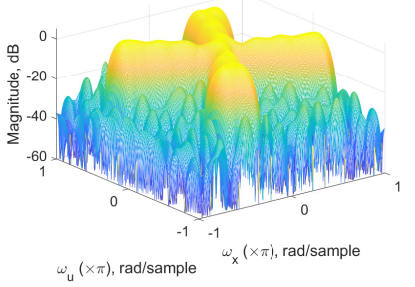

(a)

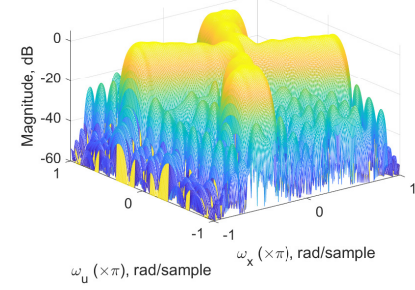

(b)
Fig. 6: Magnitude response of $H_{x u}(z)$ (a) with sparse coefficients; (b) with nonsparse coefficients.

$H_{y v}\left(z_{y}, z_{v}\right)$ as $10 \times 40$ for all the five cases. Note that the order of the resulting 4-D sparse FIR filter $H(\mathbf{z})$ is $10 \times 10 \times 40 \times 40$. We employ the CVX [28], [29] optimization toolbox to obtain the sparse impulse responses. The sparse impulse responses of $H_{x u}\left(z_{x}, z_{u}\right)$ and $H_{y v}\left(z_{y}, z_{v}\right)$ designed for the "Parc du Luxembourg" LF have 153 nonzero coefficients whereas a equivalent 2-D nonsparse FIR filter, designed with $\epsilon_{t h}=0$ in (5), has 451 nonzero coefficients. Consequently, the proposed sparse filter 4-D sparse FIR filter provides approximately $66 \%$ reduction in computational complexity compared to an equivalent 4-D nonsparse FIR filter. Fig. 6 shows the magnitude responses of $H_{x u}\left(z_{x}, z_{u}\right)$ for the sparse and nonsparse cases, and the normalized root mean square error between the two frequency responses $4.41 \%$. Considering all the five cases, the proposed 4-D sparse FIR filter provides $64 \%$ average reduction of computational complexity with a average normalized root mean square error of $4.42 \%$ compared to a nonsparse counterpart. This indicates the frequency response of the sparse filter is approximately equal to that of the nonsparse filter despite having considerably less coefficients.

Fig. 7 shows the central SAI obtained with the 4-D sparse and nonsparse FIR filters for the Parc du Luxembourg" LF. The structure similarity (SSIM) index between the central SAIs of the two refocused LFs is 0.9900 . We present the refocused central SAIs and the SSIM indices for the other four LFs in the supplementary results ${ }^{1}$. The average SSIM index between the two refocused LFs is 0.9886 . These results verify that the proposed 4-D sparse FIR filter provides negligible degradation in fidelity in multi-volumetric refocusing compared to a nonsparse counterpart.

\section{B. Comparison of the Proposed 4-D Sparse FIR Filter with Single-Volumetric-Region Refocusing Filters}

Refocusing of a single volumetric region is a special case of the proposed multi-volumetric refocusing. In this subsection, we compare the performance of the proposed filter with those proposed in [16] and [19]. To this end, we consider refocusing of LFs employed in [19]. We design the proposed 4-D sparse FIR filter with $\alpha_{1}=50^{\circ}, \theta_{1}=10^{\circ}, B_{1}=0.9 \pi \mathrm{rad} / \mathrm{sample}$, and $T_{1}=0.08 \pi \mathrm{rad} / \mathrm{sample}, \mu=0.1, \epsilon_{t h}=0.005, \gamma=1.4$, and $W\left(\omega_{x}, \omega_{u}\right)=1$ for the passband and $W\left(\omega_{x}, \omega_{u}\right)=2$ for the stopband. We also design the 4-D sparse FIR filters proposed in [19] with the same specifications for $\alpha_{1}, \theta_{1}, B_{1}$ and $T_{1}$ whereas the hard-thresholding parameter is selected as $0.03\left(h_{t h}\right.$ in [19, eq. (5)]). We design the equivalent 4-D nonsparse FIR filters [16] with the same parameters except

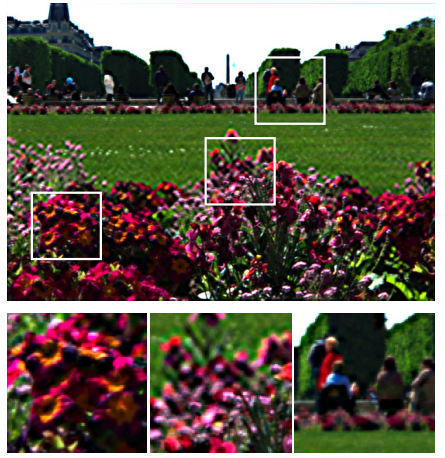

(a)

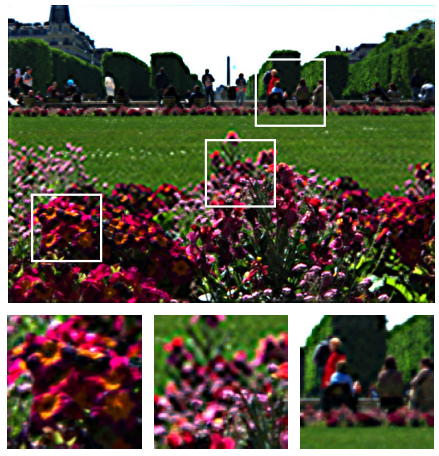

(b)
Fig. 7: "Parc du Luxembourg" LF refocused for two volumetric regions; (a) using the proposed 4-D sparse FIR filter; (b) using a 4-D nonsparse FIR filter.

TABLE I: The average SSIM indices obtained for refocusing of a single volumetric region with different 4-D FIR filters and the number of nonzero coefficients.

\begin{tabular}{l|cc}
\hline FIR Filter & SSIM index & Nonzero coefficients \\
\hline 4-D nonsparse [16] & 1.0000 & 902 \\
4-D sparse [19] & 0.9985 & 238 \\
Proposed 4-D sparse & 0.9979 & 206 \\
\hline
\end{tabular}

the hard-thresholding parameter, which is zero. We process the "Books", "Flower", "Mirabelle Prune Tree", "Sophie \& Vincent 1" and "Gravel Garden" LF with the proposed filter, the filter proposed in [19], and their equivalent nonsparse filters [16]. We present the average SSIM indices and the number of nonzero coefficients of the filters in Table I. The central SAIs of the refocused LFs are presented in the supplementary results ${ }^{1}$. According to Table I, it is evident that the proposed 4-D sparse FIR filter provides similar average SSIM index compared to the 4-D sparse FIR filter proposed in [19] while providing $13 \%$ reduction in computational complexity. The proposed 4-D sparse FIR filter achieves a lower computational complexity compared to that proposed in [19] because the former is an optimal filter whereas the latter is a sub-optimal filter. Furthermore, the proposed sparse filter provides $77 \%$ reduction in computational complexity with negligible degradation in the average SSIM index compared to an equivalent nonsparse filter [16].

\section{Conclusion}

We demonstrate simultaneous multi-volumetric refocusing of LFs by employing a 4-D sparse FIR filter consisting of multiple hyperfan-shaped passbands. We employ a two-step optimization method to design the optimal 4-D sparse FIR filters in the least-squares sense. Experimental results confirm that the proposed filter provides $64 \%$ average reduction in computational complexity with negligible degradation in the fidelity of two-volumetric-region refocused LFs compared to an equivalent 4-D non-sparse FIR filter. Furthermore, in singlevolumetric-region refocusing, the proposed filter provides $13 \%$ reduction in computational complexity compared to a previously proposed 4-D sparse FIR filters with a negligible degradation in the fidelity. 


\section{REFERENCES}

[1] M. Levoy and P. Hanrahan, "Light field rendering," in Proc. Annu. Conf. Comput. Graph. (SIGGRAPH), 1996, pp. 31-42.

[2] G. Wu, B. Masia, A. Jarabo, Y. Zhang, L. Wang, Q. Dai, T. Chai, and Y. Liu, "Light field image processing: An overview," IEEE J. Sel. Topics Signal Process., vol. 11, no. 7, pp. 926-954, Oct. 2017.

[3] S. Wanner and B. Goldluecke, "Variational light field analysis for disparity estimation and super-resolution," IEEE Trans. Pattern Anal. Mach. Intell., vol. 36, no. 3, pp. 606-619, Mar. 2013.

[4] M. W. Tao, S. Hadap, J. Malik, and R. Ramamoorthi, "Depth from combining defocus and correspondence using light-field cameras," in Proc. of IEEE Int. Conf. on Comput. Vision, 2013, pp. 673-680.

[5] T.-C. Wang, A. A. Efros, and R. Ramamoorthi, "Occlusion-aware depth estimation using light-field cameras," in Proc. of IEEE Int. Conf. on Comput. Vision, 2015, pp. 3487-3495.

[6] J. Chen, J. Hou, Y. Ni, and L.-P. Chau, "Accurate light field depth estimation with superpixel regularization over partially occluded regions," IEEE Trans. Image Process., vol. 27, no. 10, pp. 4889-4900, Oct. 2018.

[7] A. Isaksen, L. McMillan, and S. J. Gortler, "Dynamically reparameterized light fields," in Proc. Аnnu. Conf. Comput. Graph. (SIGGRAPH), 2000, pp. 297-306

[8] V. Vaish, G. Garg, E.-V. Talvala, E. Antunez, B. Wilburn, M. Horowitz, and M. Levoy, "Synthetic aperture focusing using a shear-warp factorization of the viewing transform," in Proc. IEEE Conf. Comput. Vision and Pattern Recogn., 2005, pp. 129-129.

[9] V. Vaish, M. Levoy, R. Szeliski, C. L. Zitnick, and S. B. Kang, "Reconstructing occluded surfaces using synthetic apertures: Stereo, focus and robust measures," in Proc. IEEE Conf. Comput. Vision and Pattern Recogn., vol. 2, 2006, pp. 2331-2338.

[10] D. Dansereau and L. T. Bruton, "A 4-D dual-fan filter bank for depth filtering in light fields," IEEE Trans. Signal Process., vol. 55, no. 2, pp. 542-549, Feb. 2007.

[11] C. U. S. Edussooriya, D. G. Dansereau, L. T. Bruton, and P. Agathoklis, "Five-dimensional depth-velocity filtering for enhancing moving objects in light field videos," IEEE Trans. Signal Process., vol. 63, no. 8, pp. 2151-2163, Apr. 2015.

[12] N. Liyanage, C. Wijenayake, C. Edussooriya, A. Madanayake, P. Agathoklis, L. T. Bruton, and E. Ambikairajah, "Multi-depth filtering and occlusion suppression in 4-D light fields: Algorithms and architectures," Signal Process., vol. 167, pp. 1-13, Feb. 2020.

[13] R. Ng, M. Levoy, M. Brédif, G. Duval, M. Horowitz, and P. Hanrahan, "Light field photography with a hand-held plenoptic camera," Stanford Univ., Stanford, CA, Tech. Rep. CTSR 2005-02, 2005.

[14] R. Ng, "Fourier slice photography," in Proc. Аnnu. Conf. Comput. Graph. (SIGGRAPH), 2005, pp. 735-744.
[15] J. Fiss, B. Curless, and R. Szeliski, "Refocusing plenoptic images using depth-adaptive splatting," in Proc. Int. Conf. Comput. Photogr., 2014, pp. $1-9$.

[16] D. G. Dansereau, O. Pizarro, and S. B. Williams, "Linear volumetric focus for light field cameras," ACM Trans. Graph., vol. 34, no. 2, pp. 15:1-15:20, Feb. 2015.

[17] D. G. Dansereau, D. L. Bongiorno, O. Pizarro, and S. B. Williams, "Light field image denoising using a linear 4D frequency-hyperfan allin-focus filter," in Proc. SPIE Comput. Imag. XI, vol. 8657, 2013, pp. $86570 \mathrm{P}-1-86570 \mathrm{P}-14$

[18] S. U. Premaratne, N. Liyanage, C. U. S. Edussooriya, and C. Wijenayake, "Real-time light field denoising using a novel linear 4-D hyperfan filter," IEEE Trans. Circuits Syst. I, 2020, early Access Article.

[19] S. U. Premaratne, C. U. S. Edussooriya, C. Wijenayake, L. T. Bruton, and P. Agathoklis, "A 4-D sparse FIR hyperfan filter for volumetric refocusing of light fields by hard thresholding," in Proc. IEEE Int. Conf. Digital Signal Process., 2018, pp. 1-5.

[20] W.-S. Lu and T. Hinamoto, "Two-dimensional digital filters with sparse coefficients," Multidim. Syst. Signal Process., vol. 22, no. 1-3, pp. 173 189, Mar. 2011.

[21] M. Rerabek and T. Ebrahimi, "New light field image dataset," in Proc. 8th Int. Conf. Quality Multimedia Experience, 2016, pp. 1-2. [Online]. Available: http://mmspg.epfl.ch/EPFL-light-field-image-dataset

[22] J.-X. Chai, X. Tong, S.-C. Chan, and H.-Y. Shum, "Plenoptic sampling," in Proc. Annu. Conf. Comput. Graph. (SIGGRAPH), 2000, pp. 307-318.

[23] D. Dansereau and L. Bruton, "A 4D frequency-planar IIR filter and its application to light field processing," in Proc. IEEE Int. Symp. Circuits Syst., vol. 4, 2003, pp. IV-476-IV-479.

[24] M. N. Do, D. Marchand-Maillet, and M. Vetterli, "On the bandwidth of the plenoptic function," IEEE Trans. Image Process., vol. 21, no. 2, pp. 708-717, Feb. 2012.

[25] C. Gilliam, P.-L. Dragotti, and M. Brookes, "On the spectrum of the plenoptic function," IEEE Trans. Image Process., vol. 23, no. 2, pp. 502-516, Feb. 2014.

[26] S.-C. Pei and S.-B. Jaw, "Two-dimensional general fan-type FIR digital filter design,” Signal Process., vol. 37, no. 2, pp. 265-274, May 1994.

[27] D. E. Dudgeon and R. M. Mersereau, Multidimensional Digital Signal Processing. Englewood Cliffs, NJ: Prentice-Hall, 1984.

[28] M. Grant and S. Boyd, "Graph implementations for nonsmooth convex programs," in Recent Advances in Learning and Control, ser. Lecture Notes in Control and Information Sciences, V. Blondel, S. Boyd, and H. Kimura, Eds. London: Springer-Verlag, 2008, pp. 95-110, http: //stanford.edu/ boyd/graph_dcp.html.

[29] — - "CVX: Matlab software for disciplined convex programming, version 2.1," http://cvxr.com/cvx, Mar. 2014. 